\title{
Real-space studies of the structure and dynamics of self-assembled colloidal clusters
}

\section{Citation}

Perry, Rebecca W., Guangnan Meng, Thomas G. Dimiduk, Jerome Fung, and Vinothan N. Manoharan. 2012. "Real-Space Studies of the Structure and Dynamics of Self-Assembled Colloidal Clusters." Faraday Discussions 159: 211. doi:10.1039/c2fd20061a.

\section{Published Version}

doi:10.1039/c2fd20061a

\section{Permanent link}

http://nrs.harvard.edu/urn-3:HUL.InstRepos:24902770

\section{Terms of Use}

This article was downloaded from Harvard University's DASH repository, and is made available under the terms and conditions applicable to Open Access Policy Articles, as set forth at http:// nrs.harvard.edu/urn-3:HUL.InstRepos:dash.current.terms-of-use\#OAP

\section{Share Your Story}

The Harvard community has made this article openly available.

Please share how this access benefits you. Submit a story.

\section{Accessibility}




\title{
Real-space studies of the structure and dy- namics of self-assembled colloidal clusters
}

\author{
Rebecca W. Perry, ${ }^{a}$ Guangnan Meng, ${ }^{b}$ Thomas G. Dimiduk, ${ }^{b}$ \\ Jerome Fung, ${ }^{b}$ and Vinothan N. Manoharan ${ }^{* a, b}$
}

\author{
Received Xth $X X X X X X X X X X 20 X X$, Accepted Xth XXXXXXXXX 20XX \\ First published on the web Xth $X X X X X X X X X X 200 X$ \\ DOI: 10.1039/c000000x
}

The energetics and assembly pathways of small clusters may yield insights into processes occurring at the earliest stages of nucleation. We use a model system consisting of micrometer-sized, spherical colloidal particles to study the structure and dynamics of small clusters, where the number of particles is small $(N \leq 10)$. The particles interact through a short-range depletion attraction with a depth of a few $k_{B} T$. We describe two methods to form colloidal clusters, one based on isolating the particles in microwells and another based on directly assembling clusters in the gas phase using optical tweezers. We use the first technique to obtain ensemble-averaged probabilities of cluster structures as a function of $N$. These experiments show that clusters with symmetries compatible with crystalline order are rarely formed under equilibrium conditions. We use the second technique to study the dynamics of the clusters, and in particular how they transition between free-energy minima. To monitor the clusters we use a fast three-dimensional imaging technique, digital holographic microscopy, that can resolve the positions of each particle in the cluster with $30-45 \mathrm{~nm}$ precision on millisecond timescales. The real-space measurements allow us to obtain estimates for the lifetimes of the energy minima and the transition states. It is not yet clear whether the observed dynamics are relevant for small nuclei, which may not have sufficient time to transition between states before other particles or clusters attach to them. However, the measurements do provide some glimpses into how systems containing a small number of particles traverse their free-energy landscape.

\section{Introduction}

A nucleus growing in a bulk fluid must overcome a number of challenges to become a crystal. The most well-known of these is its high surface area-to-volume ratio, which makes it prone to melting or evaporating back into the fluid. Rarely do nuclei grow to the critical size at which they are no longer unstable. A more subtle challenge arises from the structure of the nucleus, which may differ from that of the final crystal. In this case the nucleus must rearrange in order to become a bulk crystallite, and it must do so on a timescale smaller than that at which new

${ }^{a}$ Harvard School of Engineering and Applied Sciences, Harvard University, Cambridge MA 02138, USA. Fax: 1617495 0416; Tel: 1617495 3763; E-mail: vnm@seas.harvard.edu

${ }^{b}$ Department of Physics, Harvard University, Cambridge MA 02138, USA. 
particles attach. If the dynamics of rearrangement are slow, as might happen in a deeply quenched system, growth leads to metastable, disordered structures ${ }^{1,2}$.

These challenges illustrate the complex coupling between energetics, structure, and dynamics that makes nucleation a difficult process to study experimentally. Colloidal systems offer several advantages over molecular systems for such studies: the interparticle energies can be controlled using model attractive interactions such as the depletion force; the structure of the suspension can be studied in real-space, at the single-particle level, using optical or confocal microscopy ${ }^{3-5}$; and the dynamics can be made slow enough to allow the growth of nuclei to be studied in detail ${ }^{6,7}$. But even in colloids it is difficult to observe the embryonic stages of nucleation, when the nuclei are clusters rather than crystallites, and successful nucleation may hinge on a structural transition. The main source of difficulty is the disparity between the rate of cluster formation and the rate of rearrangement, which can differ by orders of magnitude. This makes it nearly impossible to find a cluster - the formation of which is a rare event that can occur anywhere in the bulk - and simultaneously observe its structural transitions. Furthermore, common three-dimensional (3D) microscopy techniques are not fast enough to image the rearrangements of a cluster on timescales short compared to the rotational and translational diffusion time of a nucleus. Thus only the late stages of growth have been investigated in 3D colloidal systems, and the early stages remain elusive.

Here we describe a different approach to addressing these challenges: we study the structure and dynamics of the clusters themselves. To avoid the problem of finding a cluster in the bulk fluid, we localize its assembly in either lithographically-prepared microwells that contain only a small number $(N \approx 10)$ of colloidal particles or by using optical tweezers to collect several particles from a dilute gas phase (Figure 1). We also use a fast 3D imaging technique, holographic microscopy, to capture the structural rearrangements of these colloidal clusters on short timescales.

These experiments do not directly probe nucleation, since the clusters are in a state of artificial isolation ${ }^{8}$ : they are either walled off from the bulk fluid or placed in a suspension too dilute to favor growth. Nonetheless, the experiments provide information critical to understanding nucleation and growth, such as the rearrangement timescales and probabilities of obtaining clusters with symmetries that differ from the bulk. A previous article ${ }^{9}$ by our group examined the energy landscape and equilibrium probabilities for small clusters $(N<10)$ in detail. Here we expand on these results by presenting (a) the chemical techniques required to control the interparticle interactions and assemble colloidal clusters; (b) a new method to image transition states and rearrangement dynamics of clusters in 3D; and (c) data on the structure and dynamics of such systems for different types of depletion interactions. Although much remains to be done to relate this type of data to bulk nucleation experiments, the results show that all three of the aspects fundamental to nucleation - energetics, structure, and dynamics - can be measured in detail through an approach combining synthesis, fabrication, and modern optical techniques.

Accepted version of Faraday Disc. 113(18): 2482 (2012) 
(a)

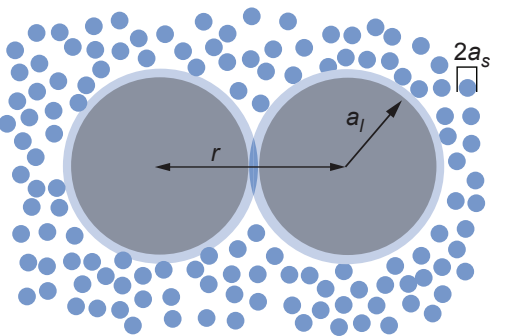

Depletion Potential

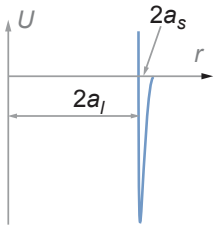

(b)

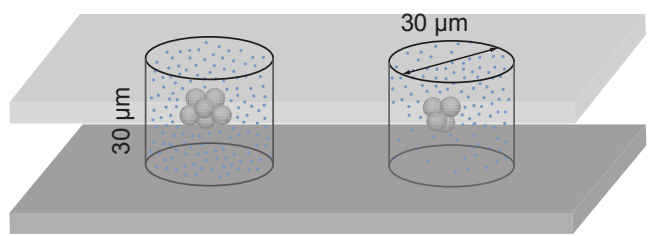

$\mathrm{N}=3$

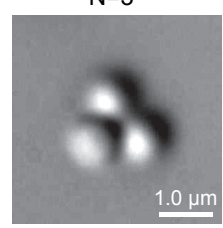

(c)
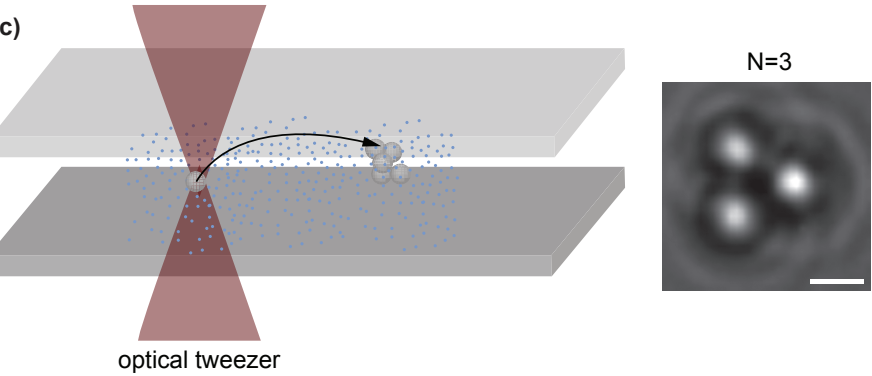

optical tweezer

Fig. 1 Experimental systems. (a) Two large spherical particles feel a mutual attraction when they come within a small sphere (depletant) diameter of each other. The width of the depletion interaction potential is much smaller than the large sphere size. (b) To self-assemble clusters of spheres, we deposit small volumes of dilute colloidal suspension into microwells. Within 24 hours, clusters form. The image at the right is an optical micrograph (differential interference contrast) of a three particle cluster. (c) A second method of assembling clusters uses an optical tweezer to bring several particles together. Once the desired number of particles is reached, the trap is turned off. The image at the right is an optical micrograph (bright field) of a three particle cluster. Cartoons of clusters in (b) and (c) are not drawn to scale. Micrograph scale bars, $1.0 \mu \mathrm{m}$. 


\section{Background}

The central theoretical concept behind our experimental study is the free-energy landscape, a multidimensional surface characterizing the free energy of a system of $N$ particles as a function of all of their configurational degrees of freedom. Understanding the landscape entails mapping out the minima, which represent stable clusters, and the transition pathways between them. Recent theoretical work has shown that the minima of the landscape can be enumerated exhaustively for a small number of hard spherical particles interacting through a short-range attraction. "Short" means that the width of the potential well is much smaller than the radius of the spheres. This limit permits a geometrical solution to the problem of enumerating the minima: the stable clusters must be rigid, or isostatic, sphere packings where the number of contacts, or "bonds," is at least $3 N-6$. To a first approximation, the potential energy of such clusters is proportional to the total number of bonds. Geometrical solutions have enumerated all possible clusters and their energies up to $N=11^{10-14}$. The transition pathways are now beginning to be enumerated through a combination of theory and simulation ${ }^{15}$.

Creating and observing clusters with such short-range interactions in an experimental system requires careful design. We work with dilute colloids to obtain the clearest possible images of clusters. To favor aggregation in such systems, the attractive interaction between colloidal particles has to be several $k_{B} T$ deep. At the same time, the binding between colloidal particles has to be reversible. If the particles become stuck together by a strong attractive potential such as the van der Waals interaction, the cluster will not be able to rearrange on experimental timescales.

We therefore use a depletion attraction, a weak entropic interaction in a binary colloidal mixture, as the driving force to assemble colloidal particles into clusters. As Figure 1(a) shows, the larger particles experience an effective attraction because the entropy of the smaller spheres, or "depletants," is maximized when the excluded volumes of the larger spheres overlap. The depletion interaction between two large spheres can be modeled by Asakura-Oosawa theory ${ }^{16,17}$ :

$$
U_{A O}(r)=-k_{B} T \frac{\pi}{6} \rho_{s}\left(2 a_{s}+2 a_{l}-r\right)^{2}\left(2 a_{s}+2 a_{l}+\frac{r}{2}\right)
$$

where $r$ is the center-to-center distance between the two large spheres, $a_{s}$ and $a_{l}$ are the radii of small and large spheres, and $\rho_{s}$ is the number density of small spheres in the solvent. The range of $U_{A O}$ is approximately the diameter of the depletants, $r<2 a_{l}+2 a_{s}$; the minimum of a purely depletion potential occurs at contact, $r=2 a_{l}$ :

$$
\begin{aligned}
U_{A O}\left(r=2 a_{l}\right) & =-\left(k_{B} T\right) \rho_{s} \frac{\pi\left(2 a_{s}\right)^{3}}{6}\left(1+\frac{3 a_{l}}{2 a_{s}}\right) \\
& \approx-\left(k_{B} T\right) 2 \pi \rho_{s} a_{s}^{2} a_{l} \quad \text { when } a_{s} \ll a_{l} .
\end{aligned}
$$

From Equation 2, when two types of colloidal particles, $1.0 \mu \mathrm{m}$ particles $\left(a_{l}=\right.$ $500 \mathrm{~nm})$ and $100 \mathrm{~nm}$ depletants $\left(a_{s}=50 \mathrm{~nm}, \phi_{s}=\rho_{S} \pi\left(2 a_{s}\right)^{3} / 6=20 \%\right)$, are mixed together, the attractive potential between large particles has a well depth of about $3 k_{B} T$ at contact and a range of $100 \mathrm{~nm}$. At very small separations, the van der Waals force might cause the large particles to stick irreversibly to one

Accepted version of Faraday Disc. 113(18): 2482 (2012) 
another, but this can be prevented by using particles with an electrostatic double layer. The range of the electrostatic repulsive barrier can be tuned through the salt concentration.

The Asakura-Oosawa model for the depletion potential assumes that the small particles reach equilibrium instantaneously as the large particles move, whereas in reality the depletion potential takes some time to saturate due to the finite diffusivity of the depletants. Using theoretical results from Vliegenthart and van der Schoot ${ }^{18}$, we estimate that for the depletants used in our study the potential saturates on timescales orders of magnitude smaller than the diffusion timescale of the large particles and the observed rearrangement timescales for our clusters (Section 4.3). Thus the kinetics of the depletants should not significantly affect the dynamics of the clusters. However, this approximation may break down for larger depletants or smaller particles.

\section{Experimental}

\subsection{Colloidal system}

Our system consists of negatively-charged polystyrene (PS) microspheres, approximately $1 \mu \mathrm{m}$ in diameter, and either poly-( $N$-Isopropylacrylamide) (PNIPAM) particles or sodium dodecyl sulfate (SDS) micelles as depletants. The sizes of the depletants are chosen so that the range of the depletion attraction is less than $10 \%$ of the diameter of the PS particles, so that the attraction is strictly pairwise additive ${ }^{19}$. Whereas the micelles are self-assembled in solution, the PNIPAM particles are synthesized beforehand and added to the suspension. In both systems the depletant scatters negligibly, allowing us to obtain clear images of the clusters through microscopy or scattering. The refractive index of PNIPAM closely matches that of our solvent, water, so the PNIPAM particles are optically transparent in aqueous solution. Furthermore, the strength of the depletion interaction can be easily controlled in both systems simply by modifying the concentration of depletants.

Using PNIPAM spheres allows us to tune the strength and range of the depletion interaction in situ. The PNIPAM depletants shrink by $50 \%$ in diameter when they are heated above their lower critical solution temperature, around $30^{\circ} \mathrm{C}$. This results in a reduction in the magnitude of the interaction strength by a factor of four, according to Equation 2.

We use precipitation polymerization ${ }^{20,21}$ to synthesize $80 \mathrm{~nm}$ PNIPAM hydrogel particles. The reactor includes a $250 \mathrm{ml}$ three-necked round bottom flask, a magnetic stirrer, a reflux condenser and a nitrogen gas inlet. We dissolve $2.0 \mathrm{~g}$ $N^{\prime}$ '-Isopropylacrylamide (NIPAM, monomer, 99\%, Acros Organics), $0.1 \mathrm{~g} N, N^{\prime}-$ Methylenebisacrylamide (BIS, crosslinker, $99 \%$, Promega), and $0.18 \mathrm{~g}(\approx 6 \mathrm{mM})$ sodium dodecyl sulfate (SDS, 99\%, EMD Chemicals) in $93 \mathrm{ml}$ DI water (Milli-Q synthesis grade, Millipore) under gentle stirring. The solution is then heated to $70^{\circ} \mathrm{C}$ and bubbled with nitrogen for one hour. To start the polymerization, we inject $40 \mathrm{mg}$ potassium persulfate (KPS, initiator, 99\%, Acros Organics) dissolved in $5 \mathrm{ml}$ DI water. During the reaction, the solution is stirred with a magnetic stirrer at $300 \mathrm{rpm}$ and bubbled with nitrogen. After four hours, the reaction is stopped by cooling the reactor down to room temperature, and the PNIPAM product is

Accepted version of Faraday Disc. 113(18): 2482 (2012) 


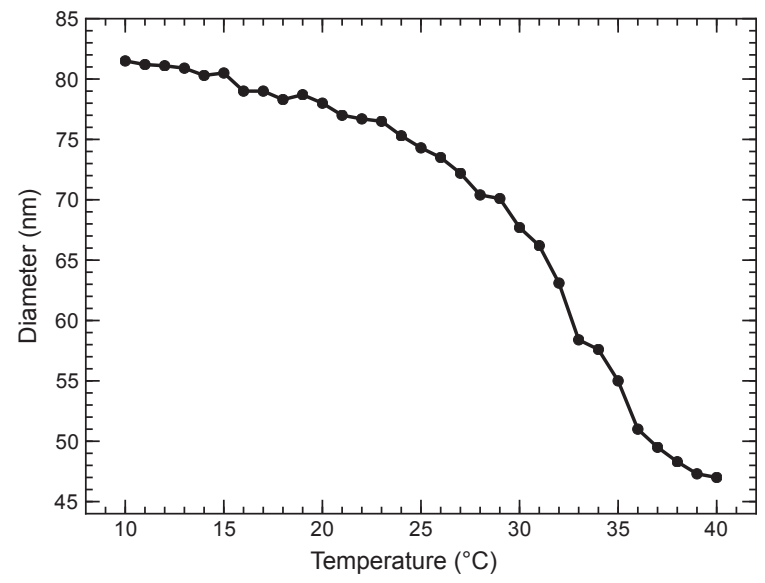

Fig. 2 Temperature dependence of the diameter of the PNIPAM hydrogel depletants.

collected. To remove unreacted monomer, initiator, and surfactant molecules from the solution, we dialyze the PNIPAM product against DI water for seven days, exchanging DI water every 24 hours.

The hydrodynamic radius of the PNIPAM particles is $80 \mathrm{~nm}$ at $20^{\circ} \mathrm{C}$ and $46 \mathrm{~nm}$ at $40^{\circ} \mathrm{C}$ with a lower critical solution temperature around $33^{\circ} \mathrm{C}$, as measured by dynamic light scattering (Zetasizer Nano ZS, Malvern Instruments) and shown in Figure 2. The polydispersity of the particles is less than 5\% at all measured temperatures. The weight concentration of particles in the stock solution is $2.13 \% \mathrm{w} / \mathrm{w}$, as measured by thermogravimetric analysis (TGA, Q5000IR, TA Instruments).

For the microwell experiments described below, we prepare a suspension of 1.0- $\mu$ m-diameter sulfate latex PS particles (Batch\# 2090,1, Invitrogen Molecular Probes, polydispersity (standard deviation in particle diameter) $3 \%$ ) and $80 \mathrm{~nm}$ PNIPAM hydrogel particles in water. For fluorescence microscopy, we use 1.0 $\mu \mathrm{m}$ sulfate fluorescent latex PS particles (FluoSpheres sulfate microspheres, 1.0 $\mu \mathrm{m}$, red fluorescent (580/605), Invitrogen, polydispersity 5\%). The volume fraction of PS particles is $10^{-5}$, and the concentration of PNIPAM is $1.0 \% \mathrm{w} / \mathrm{w}$ (estimated volume fraction $\phi_{s} \approx 0.25$ at $20^{\circ} \mathrm{C}$ ). $15 \mathrm{mM} \mathrm{NaCl}$ are added to screen the long-ranged electrostatic repulsion between the PS particles. $0.1 \% \mathrm{w} / \mathrm{w}$ Pluronic P123 (BASF) surfactant is also added to stabilize the PS particles in the salt solution. This procedure ensures that the depletion attraction between PS particles induced by PNIPAM can be reversed by either diluting the PNIPAM particles or by increasing the temperature, thereby shrinking the PNIPAM particle size.

For bulk experiments, we load the PS/PNIPAM suspension (with PS volume fraction $4 \times 10^{-3}$ ) directly into sandwiched glass cover slips through capillary action. The cover slips are separated by $40 \mu \mathrm{m}$ thick Mylar®A spacers (DuPont Teijin Films) to provide the same thickness across the samples. The edges of the glass cell are sealed with optical glue (NOA-61, Norland Products Inc.) to prevent evaporation.

For the optical-tweezer-assisted assembly method, we prepare a suspension 


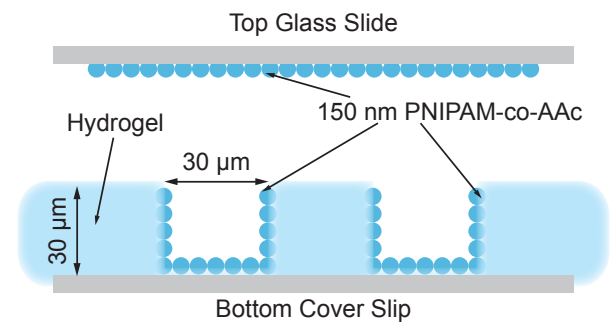

Fig. 3 Schematic of microwells and glass slides, shown from the side. All surfaces are saturated with $150 \mathrm{~nm}$ PNIPAM-co-AAc particles (not drawn to scale) to suppress the depletion attraction between the PS particles and the surfaces.

of 1.3- $\mu$ m-diameter PS particles (Batch \#1279,1, Invitrogen Molecular Probes, Surfactant-Free White Sulfate Latex, polydispersity 2.7\%), DI water, $5 \mathrm{mM} \mathrm{NaCl}$ (EMD, assay (dry basis) 99.0\%), and $40 \mathrm{mM}$ SDS (Sigma Aldrich, 99.0\%). The volume fraction of PS particles is $10^{-6}$, on the order of one particle per $100 \mu \mathrm{m}$ cube. The concentration of SDS is well above the critical micelle concentration, so the surfactant molecules assemble into micelles that act as depletants ${ }^{4}$. Following the analysis of Iracki et al. ${ }^{22}$, we estimate the width of the SDS induced depletion potential, which includes a factor proportional to the Debye length in addition to the physical size of a micelle, to be approximately $30 \mathrm{~nm}$. In terms of the width of the depletion potential, the effective micelle radius, $a_{s}$, is $15 \mathrm{~nm}$. Samples are prepared in cells consisting of a glass slide $(25 \mathrm{~mm} \times 76 \mathrm{~mm}$, VWR and a No. 1 cover slip $(22 \mathrm{~mm} \times 22 \mathrm{~mm}$, VWR). The slide and cover slip are rinsed with DI water and dried with nitrogen before use. We use UV curing epoxy (NOA-61, Norland Products Inc.) to secure $100 \mu \mathrm{m}$ thick strips of Mylar®A (DuPont Teijin Films) as spacers between the slide and cover slip. After using capillary action to fill the sample chamber with suspension, we seal the sample cell with epoxy (Devcon 5 Minute Epoxy) to prevent evaporation.

\subsection{Formation of clusters}

We prepare clusters either by letting them self-assemble in lithographically patterned microwells or by bringing particles together in a dilute suspension using an optical tweezer.

3.2.1 Microwell method. Colloidal clusters can be assembled under equilibrium conditions in microwells, as shown in Figure 1(b). Since the purpose of the microwells is to isolate a set of particles, and not to confine them, we work at small PS volume fractions such that the volume of each well is $10^{5}$ times the volume of the particles. This ensures that formation of clusters is driven by the attraction between particles rather than by a confinement effect. The solution conditions are the same for every microwell in the plate. Because each plate has tens of thousands of microwells, a single plate yields enough samples to determine ensemble probabilities of cluster structures at small $N$.

3.2.1.1 Microwell fabrication. Microwell array plates are fabricated using soft lithography ${ }^{23}$. We use standard photolithography procedures to make a mas- 
ter mold of SU-8 with the microwell pattern on the wafer. We first design a photomask pattern using AutoCAD (Autodesk Inc.). The pattern $(20 \mathrm{~mm} \times 20 \mathrm{~mm})$ has an array of circles $30 \mu \mathrm{m}$ in diameter with a pitch of $60 \mu \mathrm{m}$ on a square lattice. The pattern is printed on a photomask transparency at 20,000 dpi resolution by CAD/Art Services, Inc. We then spin coat SU-8 photoresist (SU-8 3035, MicroChem Corp.) onto a silicon wafer (University Wafer) at $3000 \mathrm{rpm}$, setting the thickness of the SU-8 layer at $35 \mu \mathrm{m}$. The microwells are made by replica molding on the SU-8 master. We prepare a pre-gel solution by dissolving $10 \% \mathrm{w} / \mathrm{w}$ acrylamide (monomer, $99 \%$, Promega), $0.5 \% \mathrm{w} / \mathrm{w} N, N^{\prime}$-Methylenebisacrylamide (crosslinker, 99\%, Promega), 0.5\% w/w allylamine (copolymer, 98\%, Alfa Aesar) and $0.1 \%$ w/w DAROCUR 1173 (photoinitiator, Ciba Specialty Chemicals Inc.) in DI water. The pre-gel solution is poured onto the SU-8 master mold and covered by a silanized cover slip (see below), which later becomes the bottom "window" of the microwell, through which the clusters can be viewed using an inverted microscope. The solution is placed $10 \mathrm{~cm}$ from an UV lamp (B-100YP, UVP) for 10 minutes to polymerize the hydrogel. The polymerized microwell plate is carefully separated from the SU-8 master mold, rinsed with DI water, and stored in DI water.

3.2.1.2 Microwell functionalization. Because the depletion attraction causes particles not only to stick to one another, but also to the walls of the microwells, we attach similar PNIPAM particles to the microwell walls and glass surfaces that bound the wells (Figure 3). This matches the roughness of the surface to the scale of the depletants, which has been shown to minimize the depletion interaction between large particles and surfaces ${ }^{24}$. We synthesize a separate batch of poly( $N$ '-Isopropylacrylamide-co-Acrylic Acid) (PNIPAM-co-AAc) hydrogel particles for this purpose. The PNIPAM-co-AAc hydrogel particles are synthesized using the same procedure as the $80 \mathrm{~nm}$ PNIPAM particles, except that we add $200 \mathrm{mg}$ acrylic acid (99\%, Sigma) to the reacting solution. The hydrodynamic diameter of these PNIPAM-co-AAc particles is $150 \mathrm{~nm}$ at $20^{\circ} \mathrm{C}$ and 60 $\mathrm{nm}$ at $40^{\circ} \mathrm{C}$. These particles are attached to the microwell boundaries and glass surfaces using silane chemistry. First the glass surfaces, either precleaned No. 1 cover slips (24 mm $\times 30 \mathrm{~mm}$, VWR) or precleaned glass slides $(25 \mathrm{~mm} \times 75 \mathrm{~mm}$, VWR), are silanized. Cover slips are silanized in 1.0\% w/w 3-Methacryloxypropyltrimethoxysilane (98\%, Sigma) in anhydrous ethanol solution for 24 hours at room temperature. Glass slides are immersed in $1.0 \% \mathrm{w} / \mathrm{w}$ (3-Aminopropyl)triethoxysilane (98\%, Sigma) in anhydrous ethanol solution for 24 hours at room temperature. Then the cover slips and glass slides are rinsed with anhydrous ethanol and blow-dried with compressed dry air. The silanization is completed by leaving the cover slips and glass slides in an oven at $110^{\circ} \mathrm{C}$ for one hour.

The cover slips form the bottom windows of the microwell plates, while the glass slides are coated with PNIPAM-co-AAc particles and used to cover the tops of the wells. To coat the microwells and silanized slides with particles, we immerse them in a dialyzed colloidal suspension of $150 \mathrm{~nm}$ PNIPAM-co-AAc particles for 24 hours at room temperature. The amine groups on the surfaces of the glass slide and acrylamide hydrogel microwells slowly bind with the carboxylic acid groups in the PNIPAM-co-AAc particles. Afterward the PNIPAM-co-AAc hydrogel particles are irreversibly adsorbed onto the surfaces. After this surface treatment, we are able to form 3D colloidal clusters of PS spheres in the middle of

Accepted version of Faraday Disc. 113(18): 2482 (2012) 
the microwells. Without the surface treatment, PS spheres form two-dimensional (2D) crystallites on the boundaries of the microwells.

3.2.1.3 Sample preparation Once the microwells are prepared and functionalized, we load them with the PS/PNIPAM suspension described in Section 3.1. The hydrogel microwell plate and glass slides are first rinsed with about $100 \mu \mathrm{L}$ PS/PNIPAM suspension at least five times, so that the hydrogel plate has the same ionic and surfactant concentration as the suspension. After the last rinse, the wells are filled with the suspension, and the microwell plate and glass slide are sealed with epoxy (Devcon 5 Minute Epoxy) around the edges of the cover slip. We find that the number of particles per well is randomly distributed with a mean of about ten. Before putting the sample on the optical microscope for observation and counting, we wait 24 hours for the system to reach equilibrium at $22.0 \pm 1.0^{\circ} \mathrm{C}$. Because the hydrogel microwells tend to deform ten days after sample preparation, the observations and data collection are done within seven days of fabrication, and the sample is discarded afterward.

3.2.2 Assisted assembly of clusters by optical tweezers. In the optical tweezer method, we start with a slide of dilute colloidal suspension and assemble a cluster one particle at a time while observing the system with an optical microscope. The microscope is equipped with an optical trap formed by an 830 nm laser (Sanyo DL-8142-201, with Thorlabs TCM1000T temperature controller and LD1255 current controller) focused through a 60X, 1.2 NA Plan Apo water immersion objective (Nikon). To build a cluster, we start by bringing two PS particles into the optical trap where they form a depletion bond. Then we add particles one-by-one to the cluster until we reach the desired $N$, as illustrated in Figure 1(c). Starting with individual particles ensures that none of the particles in the clusters are previously bonded or fused irreversibly. Once each particle is attached to the cluster by at least one bond, we turn the optical tweezer off. At this point, the cluster can explore its configurational space independent of any external potential.

\subsection{Optical methods for observation}

3.3.1 Optical microscopy. We use an inverted optical microscope (Eclipse TE-2000, Nikon Corp.) equipped with 40X dry (NA=0.9) and 100X oil-immersion $(\mathrm{NA}=1.4)$ objectives, Nomarski differential interference contrast, and epifluorescence to observe the structures of the colloidal clusters in microwells and the bulk phase behavior. A thermally insulated temperature controlled microscope stage (HSC-60, Instec Inc., with $\pm 0.1^{\circ} \mathrm{C}$ temperature stability) controls the temperature of the sample during observation. The images and videos are recorded by digital cameras $(2560 \times 1920$, Digital Sight DS-5Mc, Nikon Corp. for still images; and $720 \times 720,40$ frames per second, EO-0312C, Edmund Optics for movies) onto a personal computer. For the colloidal clusters, we scan sequentially through the microwells and record videos of clusters in each before analyzing the data.

We resolve the 3D structures of colloidal clusters by scanning through the recorded videos frame by frame. Although the microscope captures a 2D image with narrow depth of field, the rotational motion of the clusters over time allows

Accepted version of Faraday Disc. 113(18): 2482 (2012) 

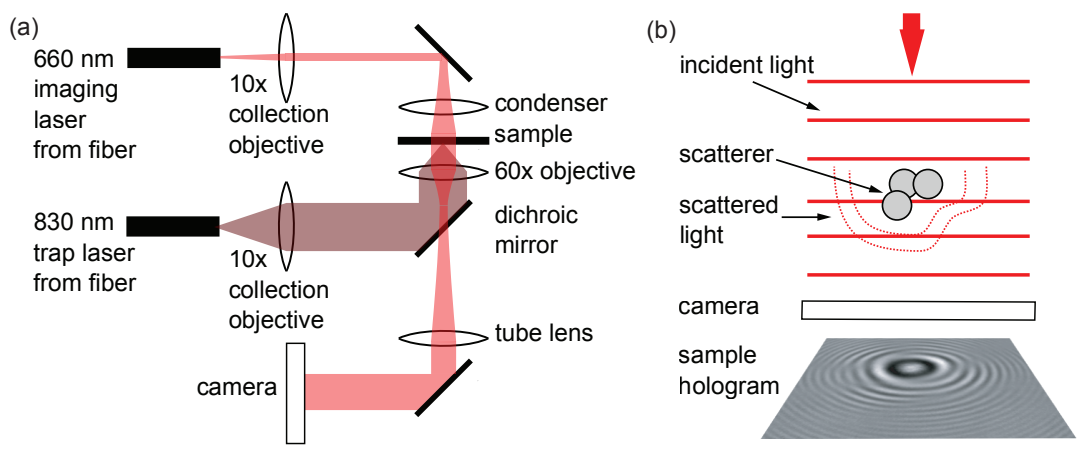

Fig. 4 Digital holographic microscopy measures the 3D positions of each particle in a cluster. (a) Schematic of apparatus. A $660 \mathrm{~nm}$ imaging laser illuminates the sample with a plane wave. A counter-propagating $830 \mathrm{~nm}$ laser creates an optical trap which is used to assemble the cluster and turned off during a dynamical measurement. (b) Diagram of hologram formation. A portion of the incident light scatters from a cluster of particles and interferes with the transmitted beam, producing a hologram that is captured by the camera.

us to see all of the particles. We map the nearest neighbors for each particle by looking at the 2D image and following it as the structure rotates in 3D space. We then compare this data to the contact matrices or computer renderings of different finite sphere packings identified in theoretical work ${ }^{10}$.

All of the micrographs shown in this paper have been subjected to linear postprocessing (brightness and contrast adjustments) to maximize clarity.

3.3.2 Digital holographic microscopy. To quantitatively image the $3 \mathrm{D} d \mathrm{dy}-$ namics of the clusters, we use digital holographic microscopy, a fast 3D imaging technique. Our apparatus consists of a Nikon Eclipse TE2000 inverted microscope modified to use a $660 \mathrm{~nm}$ laser (Opnext HL6545MG with Stanford Research Systems LDC501 laser diode current and temperature controller) for illumination, as shown in Figure 4(a). Two lenses expand and shape the laser beam so that a broad plane wave illuminates the sample as shown in Figure 4(a) and (b). The typical laser power is around $50 \mathrm{~mW}$. The light then scatters from a colloidal cluster in the sample cell (Figure 4(b)). The interference pattern of the scattered light and transmitted beam is imaged by a 60X, 1.2 NA Plan Apo water immersion objective (Nikon) and magnified by a tube lens before being recorded on a Photon Focus MVD-1024E-160-CL-12 monochrome CMOS camera. In contrast to bright field microscopy techniques, the objective is intentionally defocused so that the focal plane lies 20 to $40 \mu \mathrm{m}$ downstream of the object of interest. This allows us to better resolve the fringes in the interference pattern. We record the interference patterns at a rate of 100 frames per second with an exposure time of 15 microseconds for each frame. The images from the camera are sent through CameraLink cable to an EPIX PIXCI E4 frame grabber in a desktop personal computer, where they are recorded to disk.

As illustrated in Figure 4(b), each $256 \times 256$-pixel interference pattern (or hologram) represents the scattering from all objects in a sample volume of approximately $30 \times 30 \times 130 \mu \mathrm{m}$, centered above the objective. To remove the effects 
of scattering from irregularities on the slide or optics, we record a background image with no spheres in the field of view, normalize both it and the hologram images to have a mean value of one, and divide the holograms by the background. The normalization procedure allows us to compare our data to calculated holograms, which we also normalize to one. Background division removes irrelevant features from the data, making the interference fringes clearer.

Once the background is removed, we fit an exact scattering model to the holograms to determine the positions of the particles, following a technique originally developed for single spheres by Lee et al. ${ }^{25,26}$ and later extended to multiple spheres by Fung et al. ${ }^{27,28}$. We use a full multisphere scattering code, SCSMFO, that accounts for interference between the scattered waves, near field coupling, and multiple scattering ${ }^{29}$. This allows us to correctly fit clusters with particles separated by less than a wavelength. We use the Levenberg-Marquardt algorithm to minimize the sum of the squared residuals between a recorded hologram and a hologram calculated from the scattering model. We fit our data using the opensource software package Holopy (http://www.launchpad.net/holopy), developed in our research group.

In our procedure, we assume nothing about the cluster geometry; instead, we fit for all $3 N$ particle coordinates, plus an intensity scaling factor that accounts for variations in laser power from frame to frame. To reduce the number of free parameters in the fit, we assume a uniform particle size and refractive index. We use the particle diameter given by the manufacturer of the colloids, $1.3 \mu \mathrm{m}$, and a refractive index of $1.585^{30}$ along with a small but nonzero imaginary part of the refractive index, $0.0001 i$, to ensure that the scattering calculations converge. Once we determine the coordinates of all of the spheres by fitting, we classify the geometry of the cluster through 3D visualization or by calculating its second moment.

With $3 N+1$ parameters, the minimization problem is computationally complex. The algorithm will not converge to the actual particle positions unless we choose an initial guess for the particle positions that is close to the actual particle locations. We use two methods to generate initial guesses. The most convenient method is to use the particle positions found for the preceding or subsequent frame. This method works well at the high frame rates of our experiments, which ensure that the particles do not move far between frames. But in some cases, such as the first frame of a data series, we must guess the particle positions without any prior information. Thus we use a second method in which we determine approximate particle positions from a numerical reconstruction of a hologram ${ }^{31}$. Although near field effects prevent reconstructions from providing accurate positions of particles spaced less than a wavelength apart ${ }^{32}$, reconstructing a hologram of a lone cluster still produces an image that resembles a bright field micrograph of the cluster. By reconstructing to various planes within the sample volume, we find a plane in which the particles are approximately in focus. From this image we can estimate the relative positions and connectivity of particles in the cluster, as described in Section 3.3.1. This procedure generally yields a sufficiently precise initial guess for our fitting algorithm to converge. The resulting coordinates are then used to initialize the fit for the next frame.

To prevent the Levenberg-Marquardt algorithm from getting trapped in local minima, we allow small overlaps between particles. The algorithm either does 
not converge or converges to poor solutions when we impose a hard no-overlap condition. Instead, we allow the algorithm to place particles in positions that overlap up to $100 \mathrm{~nm}$ without any penalty. Allowing slight overlaps likely helps the fitter to avoid local minima that are due to "jammed" states, in which the most direct way for a particle to move to its true position is through another particle. Although the SCSMFO scattering calculations are not strictly defined for overlapping spheres, they nonetheless converge to within our desired numerical accuracy. When the algorithm attempts to place the spheres in positions with greater than $100 \mathrm{~nm}$ overlap, we calculate holograms of particles with reduced diameters such that the overlaps are entirely removed. Using holograms that assume the particles are smaller than their true size leads to a larger value of the objective function, effectively penalizing configurations with large overlaps. Allowing overlaps may also compensate for our assumption that the spheres are all exactly the same size.

\section{Results and Discussion}

\subsection{Interactions}

If the interactions between particles are irreversible, kinetics rather than thermodynamics will govern the structures of the clusters that assemble. Because our goal is to understand the statistical mechanics and dynamics of clusters near equilibrium conditions, we first demonstrate that the interactions between clusters are reversible and well-controlled, a necessary prerequisite to further studies.

We first examine the bulk phase behavior of PS particles at a volume fraction of $4 \times 10^{-3}$ and a constant temperature of $20^{\circ} \mathrm{C}$. At low concentrations of PNIPAM particles, $0.6 \% \mathrm{w} / \mathrm{w}$ and smaller, the PS particles remain dispersed in a gas phase, and no aggregates or crystallites form even after two weeks. As the concentration of PNIPAM increases above $0.7 \% \mathrm{w} / \mathrm{w}$, we observe quasi-2D crystals forming on the glass substrates, as shown in the optical micrographs in Figure 5. The formation of quasi-2D crystals is likely due to the depletion attraction between PS particles and the planar surface, which, unlike our microwell devices, is not treated with a layer of PNIPAM-co-AAc particles. When the surface is treated to prevent binding between the PS and the glass, we observe gelation in the bulk at a concentration of $1 \% \mathrm{w} / \mathrm{w}$ PNIPAM. The concentration dependence of the bulk phase behavior confirms that the PS particles attract one another through the depletion forces induced by the PNIPAM particles, and that the interaction can be tuned by changing the concentration of PNIPAM depletants.

At a constant concentration of PNIPAM particles, $0.8 \% \mathrm{w} / \mathrm{w}$, we find that varying the temperature from 20 to $26^{\circ} \mathrm{C}$ causes the crystals to sublimate, as shown in Figure 6. The process is reversible: after the sample is cooled to room temperature, the crystals reform. The temperature dependence is due to the change in depletant diameter on approaching the lower critical solution temperature (LCST) of the PNIPAM polymer. As shown in Equation 2, the magnitude of the depletion potential depends quadratically on the size of the depletants, $U \propto a_{S}^{2}$ for constant number density $\rho_{s}$. Since the PNIPAM hydrogel particles change their sizes from $a_{S}=40 \mathrm{~nm}$ at $20^{\circ} \mathrm{C}$ to $20 \mathrm{~nm}$ at $40^{\circ} \mathrm{C}$, the corresponding 

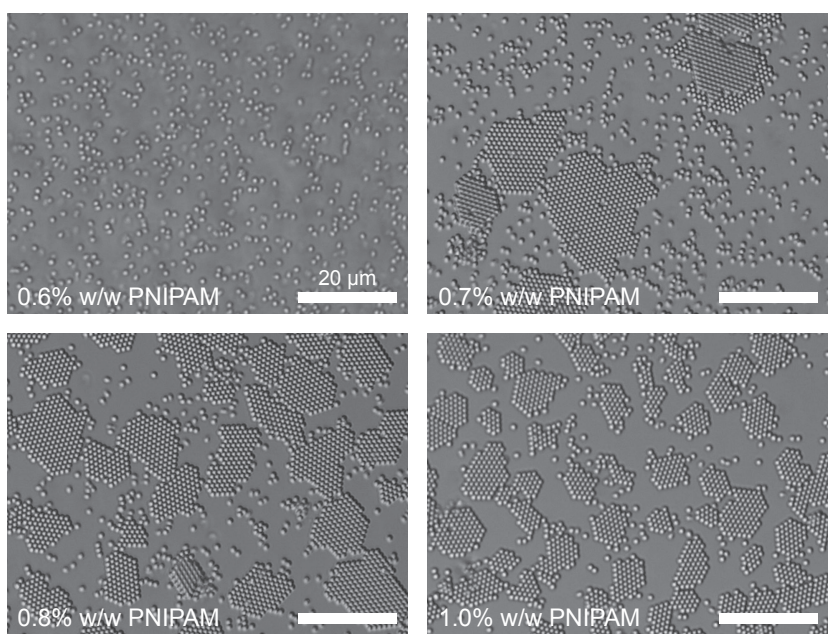

Fig. 5 Optical micrographs of $1.0 \mu \mathrm{m}$ PS colloidal particles mixed with varying concentrations of PNIPAM particles (not visible under optical microscopy). All samples are at $20^{\circ} \mathrm{C}$. PS particles form a gas phase at low PNIPAM concentration and a crystalline phase at higher PNIPAM concentration. Scale bar, $20 \mu \mathrm{m}$.

depletion potential decreases by about a factor of four over the same range. The quadratic dependence of the depletion potential on the PNIPAM size means that a relatively small change in the depletant diameter can have a large effect on the potential and can easily shift the system out of the gas-solid coexistence regime.

We observe also that the transition temperature increases with the concentration of PNIPAM hydrogel particles, in qualitative agreement with Equation 2: higher concentrations of PNIPAM increase the depletion depth, placing the system deeper into the two-phase regime, so that a larger decrease in the depletant diameter is necessary to force sublimation. Similar sublimation behavior has been observed in other systems in which the depletant size varies with temperature $^{4}$. These bulk phase behavior results show that the attraction between PS particles can be controlled over a range of a few $k_{B} T$ by changing either the concentration of PNIPAM or the temperature.

The key to achieving this kind of reversible interaction is control over the electrostatic repulsion between the PS and PNIPAM particles. At low salt concentrations, $5 \mathrm{mM} \mathrm{NaCl}$, we find that the PS particles remain dispersed as singlets even at $1.4 \% \mathrm{w} / \mathrm{w}$ PNIPAM and $10^{-2}$ volume fraction PS particles. At high salt concentration, $100 \mathrm{mM} \mathrm{NaCl}$, we observe irreversible aggregation of the PS spheres.

We also confirm that the interactions between PS particles are reversible and well-controlled when they are placed in the microwells, where we use a lower PS volume fraction. We find that at a PNIPAM concentration of $1.0 \% \mathrm{w} / \mathrm{w}$ and a PS volume fraction of $10^{-5}$, the PS particles form clusters in the middle of the microwells, with no particles stuck to the walls. As shown in Figure 7, the clusters sublimate if the temperature is increased from $25^{\circ} \mathrm{C}$ to $30^{\circ} \mathrm{C}$, indicating that the PS particles in the colloidal clusters are not trapped by van der Waals

Accepted version of Faraday Disc. 113(18): 2482 (2012) 

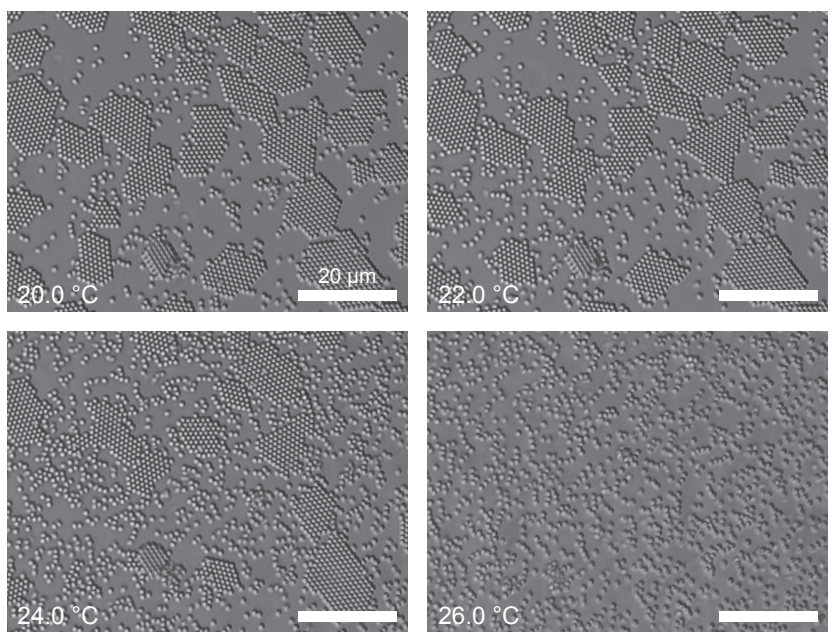

Fig. 6 Optical micrographs of a mixture of $1.0 \mu \mathrm{m}$ PS colloidal particles and $80 \mathrm{~nm}$ PNIPAM $(0.8 \% \mathrm{w} / \mathrm{w})$ particles at different temperatures. The PS particles form a crystal phase at low temperature and a gas at higher temperature. Scale bar, $20 \mu \mathrm{m}$.

forces.

Forming clusters in the microwells requires a delicate balance of the PNIPAM and PS concentrations. We choose the PS concentration to obtain the desired average number of particles per well, which is set by the microwell dimensions. Because the PS concentration is low, the PNIPAM concentration must be made high enough to overcome the tendency of the system to sublimate. Indeed, if we reduce the PNIPAM concentration slightly, to a value of $0.9 \% \mathrm{w} / \mathrm{w}$, clusters no longer form. But if the PNIPAM concentration is too high, the probabilities of formation of particular structures are biased, as shown in Figure 8. Here we plot the probability of finding a particular cluster structure, the octahedron $(N=6)$, as a function of PNIPAM concentration. The probability of forming an octahedron decreases systematically with the PNIPAM concentration (note that the error

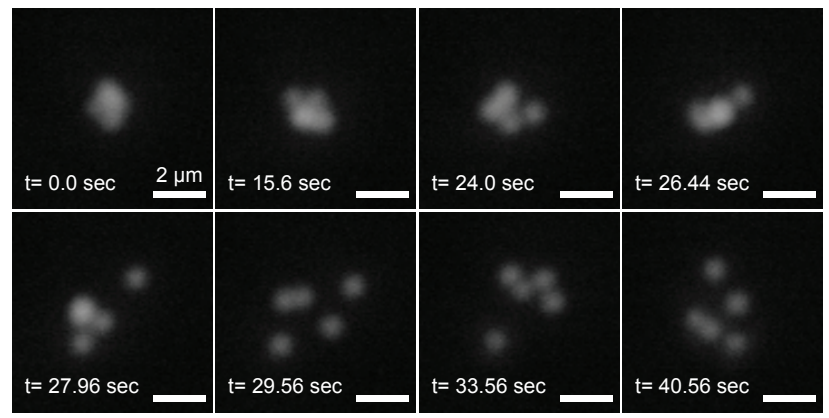

Fig. 7 Optical video microscopy snapshots of a triangular dipyramidal $(N=5)$ colloidal cluster during a sublimation transition as the temperature increases from $20^{\circ} \mathrm{C}$ to $30{ }^{\circ} \mathrm{C}$. Scale bar, $2.0 \mu \mathrm{m}$.

Accepted version of Faraday Disc. 113(18): 2482 (2012) 
bars, calculated using the Wilson score interval method ${ }^{33}$, represent $95 \%$ confidence intervals rather than standard errors on the mean). One possible source of this bias could be the variation in the depletion potential as a function of depletant concentration: a previous study showed a secondary repulsive barrier in the depletion potential at higher depletant concentrations ${ }^{34}$. The other possibility is that the formation probabilities become kinetically dominated at higher PNIPAM concentration, which corresponds to a deeper depletion well. The conditions we ultimately choose $-1.0 \% \mathrm{w} / \mathrm{w}$ PNIPAM, $10^{-5}$ volume fraction of PS particles, and $15 \mathrm{mM} \mathrm{NaCl}$ - manage to satisfy all constraints to ensure equilibrium assembly conditions.

For the dynamics experiments shown in Section 4.3, we demonstrate an alternative method of making clusters that works directly in the gas phase and does not require delicately balancing all concentrations. In these experiments we use SDS micelles instead of PNIPAM particles as the depletant, and we assemble clusters using an optical tweezer. We work at very low PS concentration, volume fraction of $10^{-6}$, and use an SDS concentration that is sufficiently large to

induce an attraction, but not large enough to cause phase separation. We test for reversible interactions by building dimers and measuring how long it takes for them to break apart. To check that the bond angles can change, we build small clusters such as bent, two-bond trimers and look for fluctuations in geometry over time. We find that $40 \mathrm{mM} \mathrm{SDS}$ and $5 \mathrm{mM} \mathrm{NaCl}$ allow us to assemble pairs of particles that remain bound for tens of seconds after the optical tweezer is turned off. This timescale is long enough to observe structural transitions in larger clusters. Under the same conditions the bond angle in a trimer can fluctuate from $180^{\circ}$ to $60^{\circ}$, corresponding to a rigid triangle. In contrast, we do not see bonds break in systems with no salt $(40-50 \mathrm{mM}$ SDS, $0 \mathrm{mM} \mathrm{NaCl})$ or too much SDS (250 $\mathrm{mM}$ SDS, $5 \mathrm{mM} \mathrm{NaCl}$ ). In such systems, rigid clusters form and are stable for more than a few minutes.

\subsection{Structures}

4.2.1 Structures of small colloidal clusters. The clusters that assemble in the microwells take on a variety of morphologies, depending on $N$. In general we find that the number of structures at each $N$ increases rapidly with $N$ for $N>6$. For each $N<6$ we observe only one structure. We observe dimers for $N=2$, triangles for $N=3$, tetrahedra for $N=4$, and triangular dipyramids for $N=5$. Following the convention in Hoy et al. ${ }^{13}$, we refer to these structures as "Barlow packings," since all of them are subsets of either a face-centered cubic (FCC) or a hexagonally close-packed (HCP) lattice.

At $N=6$, we observe two structures, an octahedron (point group $O_{h}$ ) and a "polytetrahedron" (point group $C_{2 v}$ ), which is a triangular dipyramid capped with a third tetrahedron. Optical micrographs and computer renderings in Figure 9 show the structure of these two clusters. Whereas the octahedron is a Barlow packing, the polytetrahedron is incompatible with a close-packed lattice. This is the smallest $N$ at which a non-Barlow packing occurs.

Most of the structures at $N=7$ are non-Barlow packings. We observe at least five different structures, as shown in Figure 9. In one case, we are not able to determine from the optical micrographs whether the symmetry is $C_{2 v}$ or $D_{5 h}$ (a

Accepted version of Faraday Disc. 113(18): 2482 (2012) 


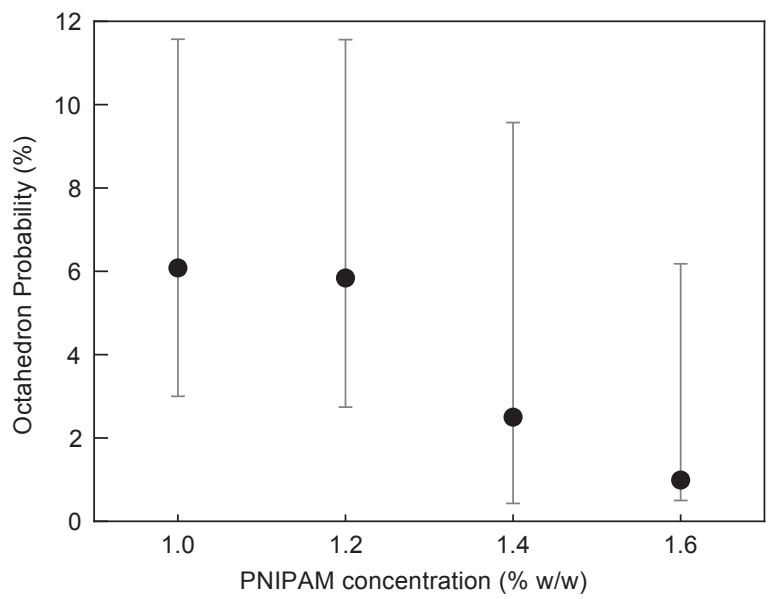

Fig. 8 Probability of observing a 6-particle octahedral cluster (black circular dots) as a function of PNIPAM depletant concentration. Error bars represent the lower and upper limits of the $95 \%$ confidence interval, as determined by the Wilson score interval method $^{33}$.

pentagonal dipyramid). For our $1 \mu \mathrm{m}$ particles, these two structures differ only in the location of a small gap of approximately $50 \mathrm{~nm}$. Two of the other structures are chiral enantiomers, both of which we observe in the measurements. Of all of these six clusters, only one, the capped octahedron with symmetry group $C_{3 v}$, is a Barlow packing.

At $N=8$, we observe at least eight different structures; again, in one case we cannot determine the symmetry, which could take on at least six possible point groups (Figure 10). All of the six possible structures are variants on the pentagonal dipyramid motif seen at $N=7$. Only two of the observed structures are Barlow packings, and both of these are derivatives of an octahedron.

All of the structures that we observe in the experiments correspond to mechanically-stable packings of hard spheres with infinitesimally short-ranged attractions. The set of all such structures up to $N=9$ was enumerated by Arkus and coworkers ${ }^{10}$. This enumeration was later extended to $N=10$ by both Arkus and coworkers ${ }^{11}$ and Hoy and O'Hern ${ }^{14}$ and recently to $N=11$ by Hoy and cowork$\mathrm{ers}^{13}$. The enumerated packings correspond to the the minima of the potentialenergy landscape as a function of $N$. Interestingly, up to $N=9$ all of these idealized packings are degenerate: they contain the same number of contacts between spheres and hence the same potential energy. The theoretical packings are shown in the renderings in Figures 9 and 10.

Three of the possible structures at $N=8$ are not observed in any of the approximately 1000 microwells we examine. These structures are annotated as " $P_{\text {exp }}=0 \% "$ in Figure 10. One of them, the gyroelongated square dipyramid (point group $D_{3 d}$ ) corresponds to a Barlow packing. It is also a derivative of an octahedron. 

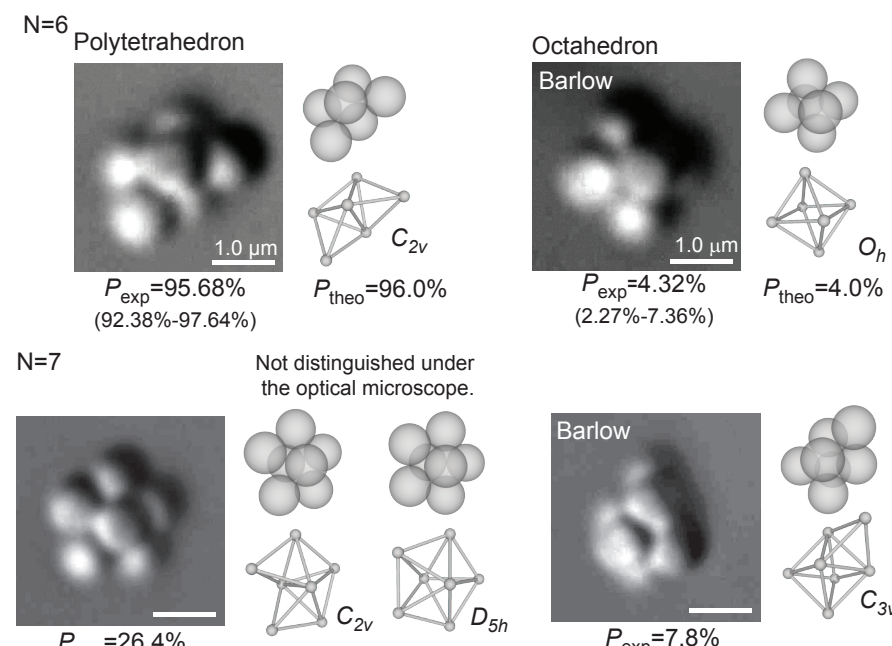

$P_{\text {exp }}=26.4 \%$

(20.5\%-33.3\%)

Not distinguished under the optical microscope.

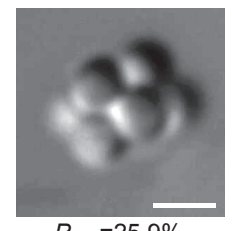

$P_{\text {exp }}=25.9 \%$ (20.0\%-32.8\%)
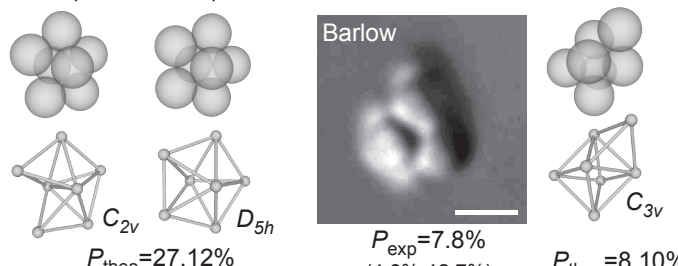

$$
\begin{aligned}
& P_{\text {exp }}=7.8 \% \\
& (4.6 \%-12.7 \%)
\end{aligned} P_{\text {theo }}=8.10 \%
$$

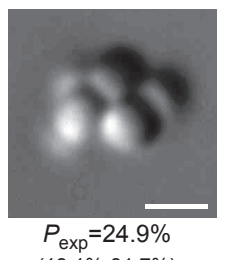
$(19.1 \%-31.7 \%)$

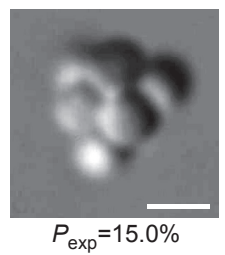
(10.5\%-21.0\%)

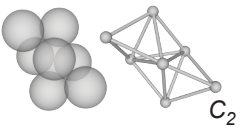

$P_{\text {theo }}=24.29 \%$

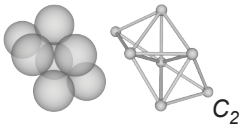

$P_{\text {theo }}=24.29 \%$

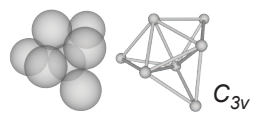

$P_{\text {theo }}=16.20 \%$

Fig. 9 Optical micrographs and renderings of colloidal clusters for $N=6$, 7, with point groups indicated in Schönflies notation. The measured (with the lower and upper limits of the $95 \%$ confidence interval determined by the Wilson score interval method ${ }^{33}$ ) and calculated probabilities are listed below each structure. Annotations above renderings indicate the clusters that cannot be distinguished under bright field microscopy.

Structures that are compatible with crystalline lattices are marked with "Barlow". Scale bar, $1.0 \mu \mathrm{m}$. 
$\mathrm{N}=8$
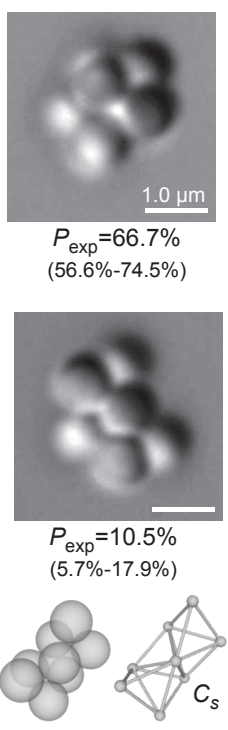

$P_{\text {theo }}=9.28 \%$

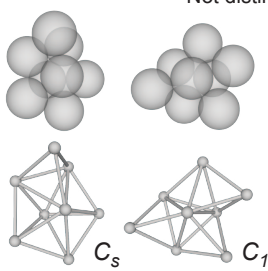

Not distinguished under the optical microscope.
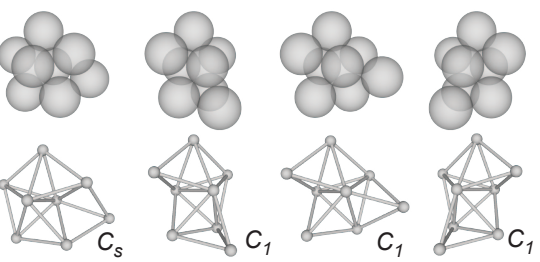

$P_{\text {theo }}=51.44 \%$
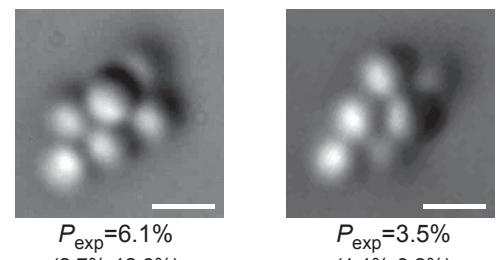

$P_{\text {exp }}=3.5 \%$ $(2.7 \%-12.6 \%)$

$(1.1 \%-9.2 \%)$
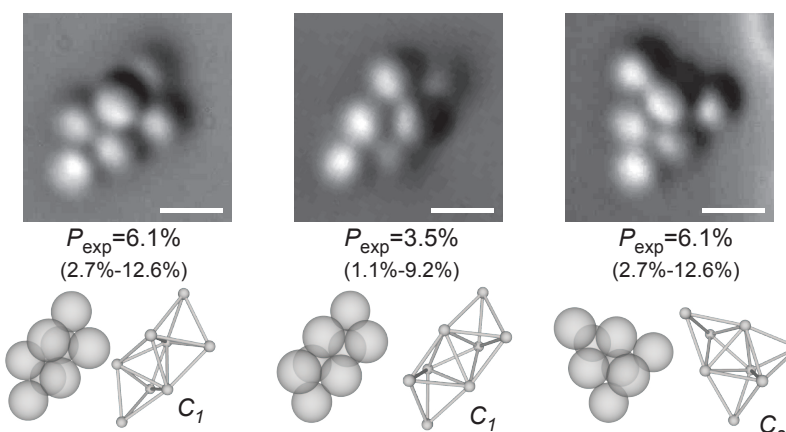

$P_{\text {theo }}=9.28 \%$

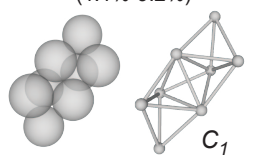

$P_{\text {theo }}=9.28 \%$
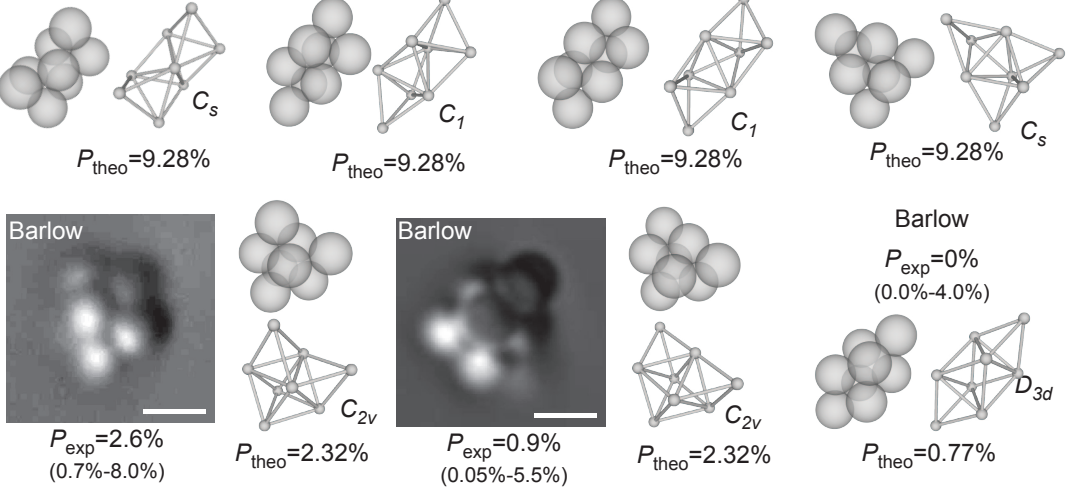

$(0.7 \%-8.0 \%)$
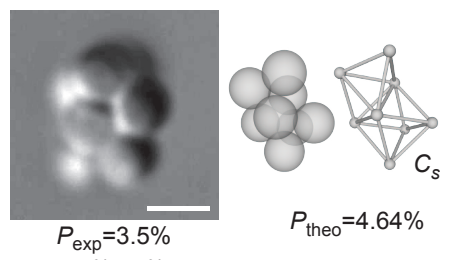

$P_{\text {theo }}=4.64 \%$
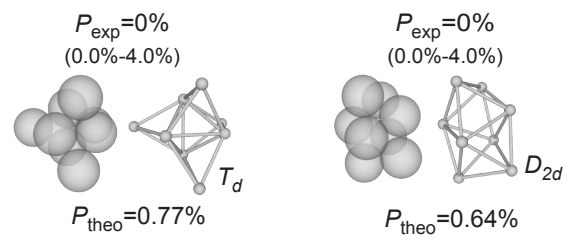

Fig. 10 Optical micrographs and renderings of colloidal clusters for $N=8$, with point groups indicated in Schönflies notation. The measured (with the lower and upper limits of the $95 \%$ confidence interval determined by the Wilson score interval method ${ }^{33}$ ) and calculated probabilities are listed below each structure. Annotations above renderings indicate the clusters that cannot be distinguished under bright field microscopy.

Structures that are compatible with crystalline lattices are marked with "Barlow". Scale bar, $1.0 \mu \mathrm{m}$.

Accepted version of Faraday Disc. 113(18): 2482 (2012) 
4.2.2 Probabilities and free energies. We measure the free energy of each of the cluster structures simply by counting the number of occurrences of each cluster on the microwell plate. If the clusters are in equilibrium, the distribution of cluster structures should follow the Boltzmann distribution, $F_{i} \propto-k_{B} T \ln \left(P_{i}\right)$, where $P_{i}$ is the probability of observing structure $i$. For example, at $N=6$, we observe about $4 \%$ octahedra and $96 \%$ polytetrahedra, implying that the free energy of a polytetrahedron is about $3 k_{B} T$ lower than that of an octahedron. This difference can be attributed only to entropy, since the two structures have the same number of contacts between particles - or "bonds" - and hence the same potential energy. The measured probabilities for each structure are shown in Figures 9 and 10 . Where it is not possible to determine the particular symmetry group of a cluster from the micrographs, we add together the probabilities of all possible structures.

As we showed in previous work ${ }^{9}$, all the measured probabilities agree well with theoretical calculations for the rotational and vibrational entropies. Both sets of probabilities are shown in Figures 9 and 10. The dominant contribution to the free energy comes from the rotational entropy: structures with higher rotational symmetry are much less likely to form than less-symmetric structures. This is because the symmetry number of a structure is inversely related to the number of permutations of particles that do not change the structure ${ }^{15}$. Each permutation corresponds to a different pathway to the same structure, and in equilibrium, all such pathways are equally probable ${ }^{8}$. We note that at $N=8$, the three structures we do not observe have high symmetry, and thus low probability $\left(P_{\text {theo }}<1.0 \%\right)$ of formation.

In terms of nucleation, the most striking feature of the results up to $N=8$ is the low probability of forming a structure compatible with a close-packed lattice. The total probability of all possible Barlow packings is about $4 \%$ for $N=6$, $8 \%$ for $N=7$, and $5 \%$ for $N=8$. The most likely structures are the least symmetric ones, which in general correspond to packings based on a polytetrahedral motif ${ }^{35}$. Hoy et al. ${ }^{13}$ found similar probabilities in their theoretical study.

The situation becomes more complicated when there are more than 9 particles in a cluster: at $N=9$, clusters with soft modes first appear, and at $N=10$ clusters with greater than $3 N-6$ bonds can form. These structures, many of which are Barlow packings, occur frequently in the experiments, as shown in Figure 11. This result is qualitatively in agreement with theory: vibrational entropy associated with soft modes stabilizes the non-rigid clusters, while the potential energy associated with the extra bond stabilizes the clusters with $3 N-5$ bonds. Quantitative agreement is more difficult to obtain, since an accurate theoretical calculation of the free energy of the non-rigid clusters requires detailed knowledge of the pair potential. This is because the soft modes dominate the vibrational entropy, and the amplitude of these modes depends on the curvature of the potential near its minimum. Since the probabilities of the non-rigid clusters are non-negligible, any error will also affect a calculation of the probability of forming a cluster with extra bonds.

If at larger $N$ there is a similar correlation between Barlow packings and extra bonds or soft modes - as we expect there might be, since structures with extra bonds and soft modes tend to contain both octahedral and tetrahedral subunits ${ }^{11}$, a necessary precondition for an FCC or HCP substructure - then there could be 

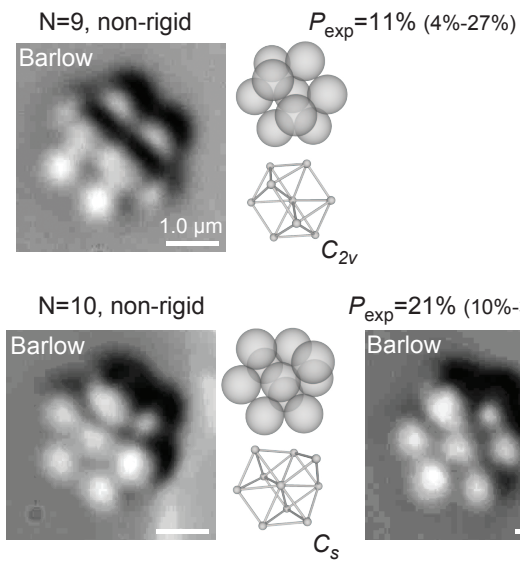

$P_{\text {exp }}=21 \%(10 \%-39 \%)$
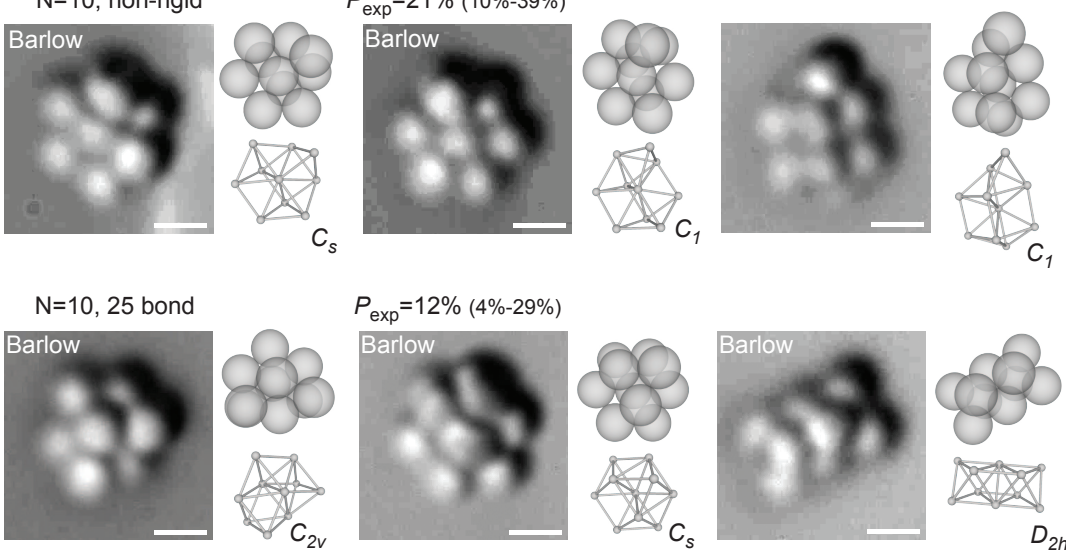

Fig. 11 Optical micrographs and renderings of special colloidal clusters for $N=9$ and $N=10$, with point groups indicated in Schönflies notation. The measured (with the lower and upper limits of the $95 \%$ confidence interval determined by the Wilson score interval method ${ }^{33}$ ) probabilities are listed above the categories. Structures that are compatible with crystalline lattices are marked with "Barlow". Scale bar, $1.0 \mu \mathrm{m}$.

a significant implication for nucleation in similar kinds of short-range attractive systems: the probability of forming a Barlow cluster would depend not only on the potential depth, but also on the curvature of the potential, or its spring constant.

\subsection{Dynamics}

The microwell experiments highlight the low probabilities of forming Barlow packings at low $N$. Even for $N \geq 9$ the probabilities do not exceed 25\%, although, as we have noted, these results may depend on the details of the potential. Connecting these results to nucleation barriers in bulk systems requires understanding the internal dynamics of the clusters. As Crocker noted ${ }^{8}$ about Meng et al.'s original experiments ${ }^{9}$, our "clusters can equilibrate at leisure in complete isolation, [whereas] the clusters in an unbounded fluid are continuously bombarded by and grow by absorbing smaller clusters..., all of which may frustrate the equilibration of internal modes." While growth has been well characterized ${ }^{36}$, little is known about the second process, internal equilibration. The rate-limiting step for equilibration is rearrangement between cluster structures, an activated process that requires breaking at least one bond. Here we examine the dynamics of rearrangements in real space. 

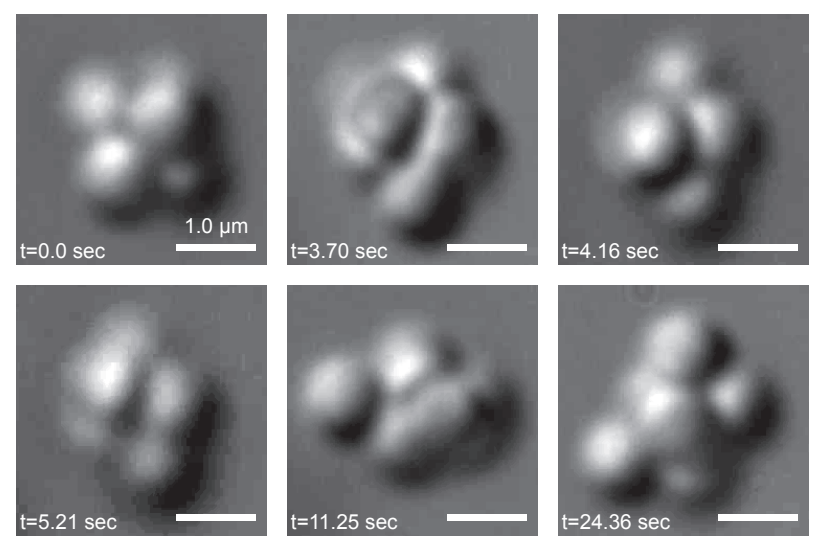

Fig. 12 A transition between an octahedral and polytetrahedral cluster in a microwell, captured using optical microscopy. Scale bar, $1.0 \mu \mathrm{m}$.

4.3.1 Dynamics of transitions under bright field microscopy. We find that our clusters can and do transition between different structures after formation. For the PNIPAM system, we find that a six particle cluster changes its structure from a polytetrahedron to an octahedron and back every few minutes to tens of minutes. A typical transition as viewed through optical microscopy is shown in Figure 12. The transition itself occurs on a timescale of seconds. We observe similar transitions at larger $N$.

The short timescale of the transition makes it difficult to determine the structure of the transition state. When a cluster is in an energy minimum, we can infer the relative positions of all the particles because the rotational Brownian motion of the cluster eventually brings all the particles within view. In contrast, the lifetime of a transition state is significantly shorter than the timescale of rotational motion, so we can only obtain qualitative data on the transition-state structures. For example, the micrographs in Figure 12 appear to show that one of the twelve bonds breaks, and a new bond forms between different particles, but we cannot confirm this without quantitative measurements of the 3D positions of all six particles, accurate to $100 \mathrm{~nm}$ or better. Further complicating measurements of the dynamics is the long lifetime of the minima relative to that of transition states. Transitions are therefore rare events, and capturing just one of them may require recording tens of thousands of frames.

We therefore use a different experimental technique and, at the same time, modify our system to make it possible to study the dynamics of the clusters. To image the clusters we use holographic microscopy instead of optical microscopy. Holographic techniques can resolve the positions of all the particles in a cluster with at least $100 \mathrm{~nm}$ precision and $10 \mathrm{~ms}$ temporal resolution. We also change the depletant from PNIPAM particles to SDS micelles, and we assemble clusters directly in the gas phase using an optical tweezer. Although the clusters obtained in this way are thermodynamically unstable after the tweezer is turned off, they survive long enough to allow us to study transitions, as noted in Section 4.1. Also, the rate of transitions is higher than in the PNIPAM-microwell system. The reasons for this are not clear, but the simplest explanation may be that the potential

Accepted version of Faraday Disc. 113(18): 2482 (2012) 
well is not as deep. In the microwell system we must use a deep potential well to force the particles to aggregate at low concentration. In experiments where we manually concentrate particles using an optical tweezer, we are free to tune the depletant concentration to optimize the kinetics. SDS micelles are more convenient than PNIPAM particles for this purpose because they are much simpler to make and mix. They also lead to a similar "sticky" depletion potential, in which the range of the attraction is much smaller than the diameter of the PS particles.

4.3.2 Validation of holographic microscopy technique. Because holographic microscopy has not previously been used to study dynamics of clusters larger than two particles, we first show that our fitting method yields realistic and accurate particle positions. Since a hologram is a 2D encoding of a 3D system and not simply a projection, we cannot verify the calculated particle coordinates by overlaying them on top of a real-space image, as one might do in standard particle tracking techniques based on optical microscopy ${ }^{37}$. Instead, we verify the calculated coordinates by numerically comparing measured holograms to ones obtained by fitting a scattering solution to the data ("best-fit holograms"). We also compare numerical reconstructions of the measured and best-fit holograms.

An example of the results obtained from our method is shown in Figure 13(a) for one of the more complicated holograms to fit, one taken of a six-particle cluster that has formed an octahedron. Qualitatively, the data and the hologram calculated from the fit appear identical: the interference rings are in the same locations, and the deviations from a circular symmetry are in the same places. Quantitatively, the model fits the data well. The mean of the squared residuals across all pixels, $\chi^{2} \approx 4 \times 10^{-4}$, is within a factor of 10 of the noise floor for the measured holograms, $5 \times 10^{-5}$. This corresponds to an uncertainty in the particle positions of $30-45 \mathrm{~nm}$ in $x, y$, and $z$, consistent with previous findings ${ }^{27}$.

To further verify the accuracy of the fit, we reconstruct both the measured and best-fit hologram, as shown in Figure 13(b). To generate these reconstructions we numerically propagate light through the hologram to the midpoint of the cluster, as determined by the fit. Although the reconstructions do not account for coupling between the scattered fields of the particles, they nonetheless reveal an approximate image of the cluster. The cluster structure and orientation suggested by the reconstructions agree well with those computed from the fit, as shown in Figure 13(c).

4.3.3 Dynamics of small clusters. Having demonstrated that fitting exact scattering solutions to holograms reveals accurate cluster structures, we now examine the measurements of cluster dynamics obtained from time-series of holograms. We fit for all $3 \mathrm{~N}$ particle coordinates as a function of time, but for simplicity we characterize the cluster structure by an order parameter $M_{2}$, the second moment of the mass distribution ${ }^{38}$ :

$$
M_{2}=a_{\mathrm{eff}}^{-2} \sum_{i=1}^{N}\left|\mathbf{r}_{i}-\mathbf{r}_{0}\right|^{2}
$$

$\mathbf{r}_{i}$ is the location of the $i^{t h}$ sphere, $\mathbf{r}_{0}$ the center of mass of the cluster, and $a_{\mathrm{eff}}$ the effective radius of the particles, or half the distance from the center of one particle, across the depletion zone, to the center of a neighboring particle. We take $a_{\mathrm{eff}} \approx a_{l}+a_{s} / 2$ and $a_{s} \approx 15 \mathrm{~nm}$ for an SDS micelle.

Accepted version of Faraday Disc. 113(18): 2482 (2012) 
(a)

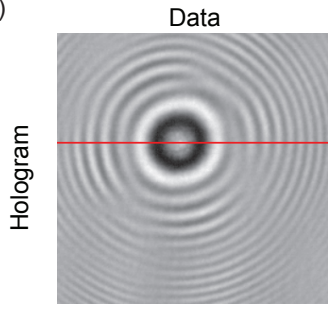

(b)

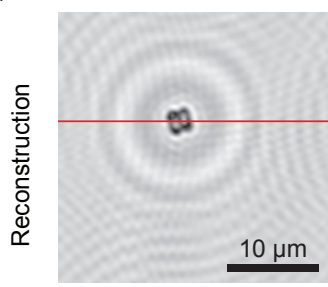

(c)

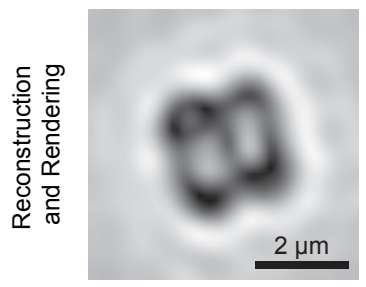

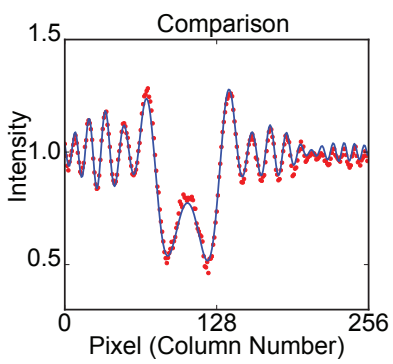
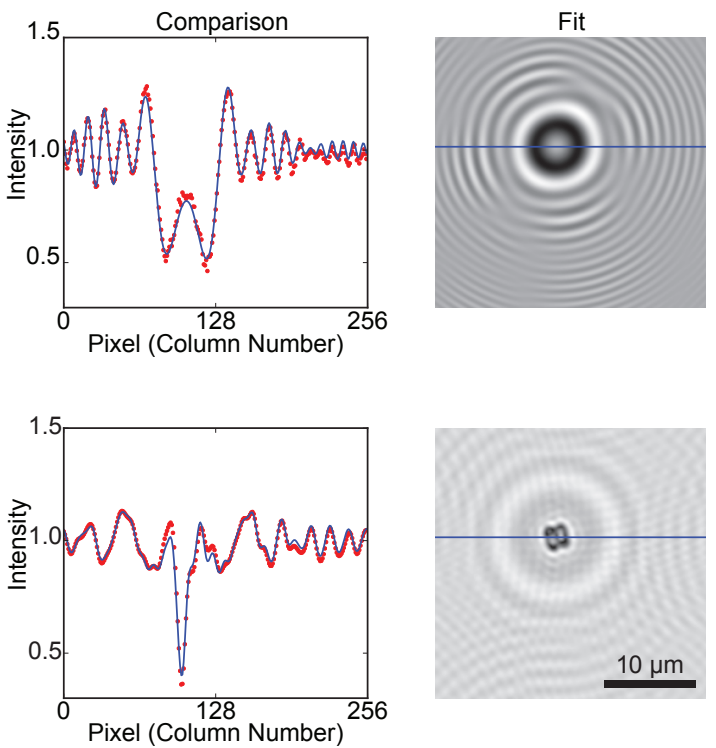

$10 \mu \mathrm{m}$
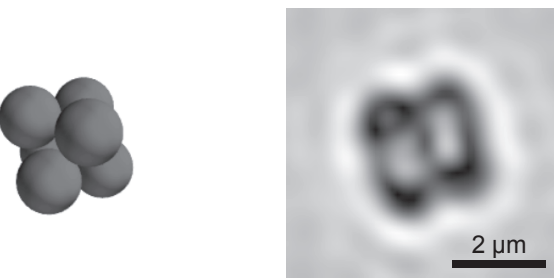

Fig. 13 Fitting holographic microscopy data to exact scattering models reveals the locations of all particles in 3D. (a) A frame of raw holographic data for a 6-particle cluster (left) and the hologram calculated from the best-fit positions of the particles (right). Middle plot shows a comparison between the intensities of the two holograms along the linear cross-sections shown in the images. Dotted line corresponds to the measured hologram and solid line to the best-fit hologram. (b) Holographic reconstructions of the raw data (left) and of the best-fit hologram (right). As above, middle plot shows a comparison between intensities of the two images across a linear cross-section. Scale bars, $10 \mu \mathrm{m}$. (c) Close-ups of the two reconstructions along with a rendering of the octahedral cluster generated from the fitted particle locations, showing that the fit agrees qualitatively with the reconstructed images. Scale bars, $2 \mu \mathrm{m}$. 


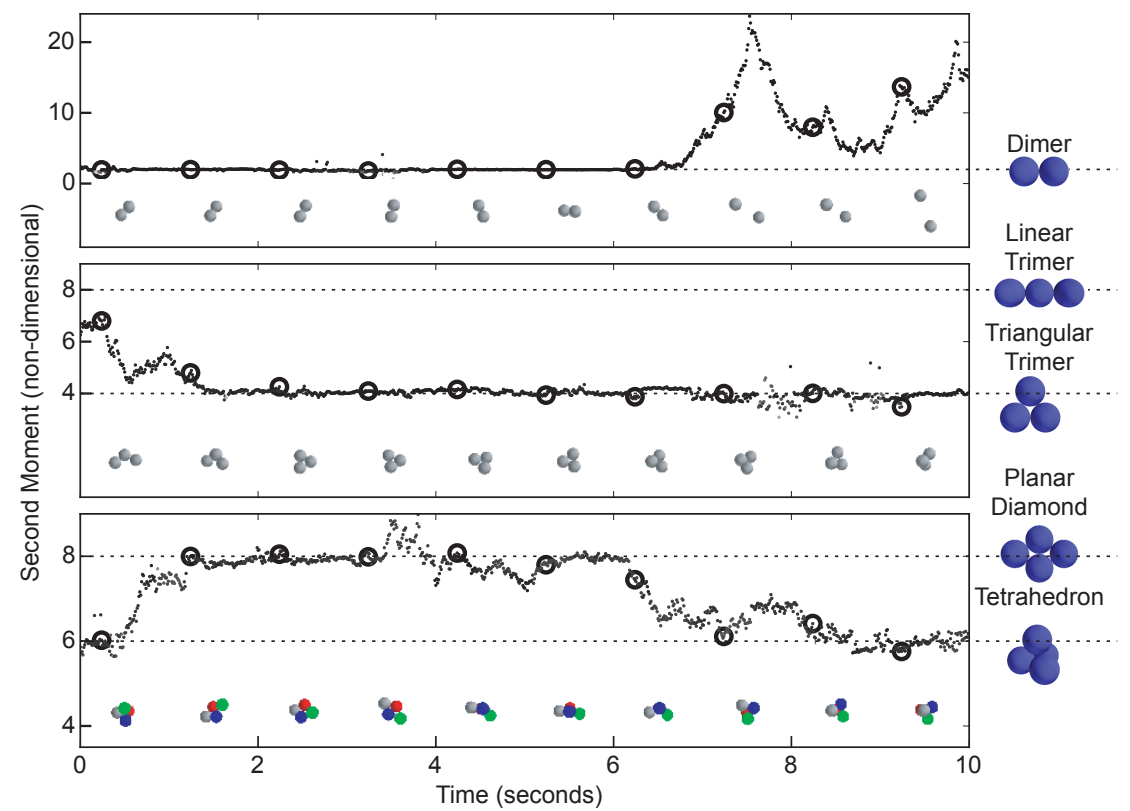

Fig. 14 Cluster dynamics as determined by holographic microscopy for 2-, 3-, and 4-particle clusters. Plots show the second moment $M_{2}$ (Equation 3) as a function of time. The intensity of the data points indicates the relative value of the goodness-of-fit parameter $\chi^{2}$ (black represents the lowest $\chi^{2}$ ). Dotted lines show $M_{2}$ for the reference geometries at right $($ Dimer $=2$, Linear Trimer $=8$, Triangle $=4$, Planar Diamond $=8$, Tetrahedron $=6$ ). Renderings within each plot show the cluster configurations corresponding to the nearest circled data points. 
The variation in cluster structure with time, as characterized by $M_{2}$ and realspace renderings of the cluster coordinates, is shown in Figure 14 for 2-, 3-, and 4-particle clusters. Although such clusters have only a single free-energy minimum, as discussed in Section 4.2.1, they show transitions between rigid and non-rigid states as well as rotational and translational Brownian motion. We see a dimer $(N=2)$ break apart, a trimer $(N=3)$ assemble itself into a rigid triangle, and a tetramer $(N=4)$ transition from a tetrahedron to a planar diamond and back to a tetrahedron. Interestingly, the tetrameric transition is an inversion: labeling the particles shows that the handedness of the tetrahedron changes from the beginning to the end of the measured trajectory (see color renderings in Figure 14). Similar types of tetrahedron-diamond-tetrahedron transitions may occur in larger clusters, where they could represent a mechanism for isomerization between different polytetrahedral configurations.

The data show that the lifetime of a non-rigid state is on the order of seconds. To understand this lifetime we estimate the timescale for the tetrahedrondiamond-tetrahedron inversion. In this transition, one of the end particles must traverse an arc length of approximately $110^{\circ}$. Neglecting translations of the center of mass and global rotations, the path length this end particle must travel is $\frac{110}{180} \pi \sqrt{3} a_{l}=2.2 \mu \mathrm{m}$, since it is $\sqrt{3} a_{l}$ from the rotation axis. Using the diffusion coefficient for a single particle $D=3.1 \times 10^{-13} \mathrm{~m}^{2} / \mathrm{s}$, which we measure in a separate experiment by holographically tracking an unbound particle, we estimate that the rearrangement should occur in about 7 seconds, which is close to the lifetime we observe. The agreement between the calculation and data shows that the lifetime of the non-rigid state is likely diffusion-limited, and that there are no significant hydrodynamic corrections to the diffusion time for bond rotation.

4.3.4 Dynamics of a transition between two free energy minima. As described in Section 4.2.1, a six particle cluster is the smallest cluster that can transition between two rigid energy minima: an octahedron, which is a Barlow packing, and a polytetrahedron, which is not. Using holographic microscopy, we observe a six particle cluster form a polytetrahedron and transition to an octahedron. The results, summarized in Figure 15, contain far more detail than can be obtained from the bright field micrographs in Figure 12.

The ball-and-stick renderings of Figure 15 show the bonds that form and break during the transition. Initially, there are only 10 bonds between the six particles. Four of the particles, shown in gray, are bound in a rigid tetrahedron. Shortly after $t=2 \mathrm{~s}$, the particle labeled in blue bonds to the tetrahedron to form a trigonal dipyramid. Then an additional particle, shown in red, bonds to the dipyramid to complete the formation of a polytetrahedron at around $t=3 \mathrm{~s}$. Just before $t=6 \mathrm{~s}$, a bond breaks, and the cluster rapidly transitions to an octahedron, which persists until the end of the data set.

The observed timescale for this transition, which transforms the cluster from a structure inconsistent with crystallinity to a Barlow packing, is close to the timescale expected from single-particle diffusion. This transition requires two particles to move from $31 / 3$ radii apart to 2 radii apart in order to form a bond. For simplicity, we consider only the time it takes one particle to diffuse a linear distance of $4 a_{l} / 3$. From the measured single particle diffusivity, this should take approximately one second, consistent with our observations.

We can estimate the rate of transitions from the observed structure lifetime,

Accepted version of Faraday Disc. 113(18): 2482 (2012) 


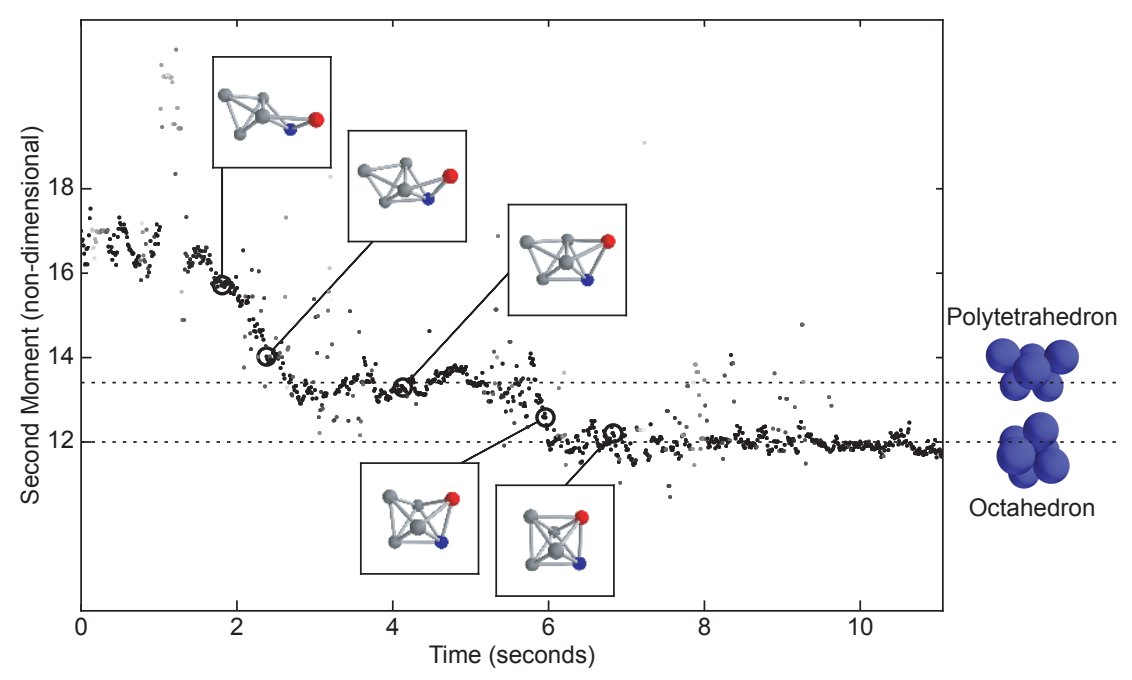

Fig. 15 Transition from polytetrahedron to octahedron in a 6-particle cluster, as measured by holographic microscopy. Plot shows evolution of the second moment $M_{2}$ as a function of time, and ball-and-stick insets show the cluster geometry. The insets are oriented to clearly show the cluster structure and do not represent the actual spatial orientation of the clusters. Dashed lines indicate the second moments of the polytetrahedron $\left(13 \frac{11}{27}\right)$ and the octahedron (12).

which is also on the order of 1 second. We assume that it is equally likely for any of the 12 bonds in the polytetrahedron to break. Only one of these breakages can lead to the formation of an octahedron; the other 11 will result in tetrahedrondiamond-tetrahedron transitions that do not change the structure. Thus we expect that transitions from polytetrahedra to octahedra should in general happen on timescales of tens of seconds; presumably we were fortunate to be able to capture, in our short data set, the breaking of the one bond that would allow an octahedron to form. For comparison we estimate the growth rate as a function of the volume fraction, assuming diffusion-limited conditions. At $10^{-6}$ volume fraction, new particles arrive at the cluster every hour, at $10^{-4}$ every few minutes, and at $10^{-2}$ every few seconds, which is comparable to the time between structural transitions.

The $N=6$ cluster is the smallest system in which growth can lead to two different outcomes: Barlow packing or polytetrahedral order. A new particle that attaches to an octahedron produces another Barlow packing, while one that attaches to a polytetrahedron produces an $N=7$ polytetrahedron. At higher volume fractions, when the growth rate is comparable to the transition rate, we might expect that the system has a greater tendency to develop polytetrahedral order, which is incompatible with crystal nucleation. Given the low free energy of the $N=6$ polytetrahedron relative to that of the octahedron, the prospects for successful nucleation of a crystal from an $N=6$ embryo seem bleak. However, at higher volume fraction the initial clusters that form may be much larger than six particles, so the $N=6$ case may not in general represent a nucleation "bottleneck." Further studies of transitions in larger systems, where extra bonds and soft 
modes are possible, are necessary to more rigorously relate the cluster dynamics to nucleation probabilities.

\section{Conclusions}

The work we have shown here represents the first steps toward understanding nucleation through analysis of the thermodynamics and dynamics of colloidal clusters. Much remains to be done on both the experimental and theoretical fronts, particularly for larger clusters. Also, although we have measured transition rates for a few small clusters, we need much more data on both small and large clusters to obtain statistically significant estimates of the transition state lifetimes and transition pathways, which are the key elements missing from the free-energy landscape model of short-range attractive spheres ${ }^{9,15}$. Such studies will require measurements of the interaction potential: as we have shown, the rearrangement timescales for systems with different depletants can vary by orders of magnitude, presumably because of differences in the well depth and width; also, the probabilities of obtaining clusters with soft modes, many of which are Barlow packings, depend on the curvature of the potential and not just the well depth. To measure these features of the potential we must be able to resolve the separation between two colloidal particles to nanometer-scale precision. This is a difficult task, but recent advances in imaging ${ }^{27}$ and optical-tweezer-based measurements ${ }^{39}$ show that it is possible.

Although the connection to nucleation barriers remains tenuous at this stage, our work demonstrates that the study of colloidal clusters stands to reveal new insights into processes that are key to understanding nucleation, including the formation of clusters and their structural transitions. Modern experimental techniques such as soft lithography and holographic microscopy make it possible to measure all the thermodynamic and dynamical information about a cluster, including its structure, free energy, and fluctuations about free-energy minima. We know of no other experimental system that can be probed in such detail. The main goal for future experiments is to systematically explore the dynamics as a function of $N$ and to obtain, from that wealth of detail, a more complete model of the free-energy landscape that governs nucleation.

\section{Acknowledgements}

We thank Jesse Collins, Miranda Holmes-Cerfon, Zorana Zeravcic, Natalie Arkus, Michael Brenner, and Robert Hoy for helpful discussions. Rebecca W. Perry and Thomas G. Dimiduk acknowledge the support of National Science Foundation (NSF) Graduate Research Fellowships. This work was funded by the NSF through CAREER grant no. CBET-0747625 and through the Harvard MRSEC, grant no. DMR-0820484. Computations were performed on the Odyssey cluster, managed by the Harvard FAS Sciences Division Research Computing Group. Microwell device fabrication was performed in part at the Center for Nanoscale Systems (CNS), a member of the National Nanotechnology Infrastructure Network (NNIN), which is supported by the NSF under award no. ECS-0335765. CNS is part of Harvard University.

Accepted version of Faraday Disc. 113(18): 2482 (2012) 


\section{References}

1 V. J. Anderson and H. N. W. Lekkerkerker, Nature, 2002, 416, 811-815.

2 C. P. Royall, S. R. Williams, T. Ohtsuka and H. Tanaka, Nature Materials, 2008, 7, 556-561.

3 W. K. Kegel and A. van Blaaderen, Science, 2000, 287, 290-293.

4 J. R. Savage, D. W. Blair, A. J. Levine, R. A. Guyer and A. D. Dinsmore, Science, 2006, 314, 795-798.

5 P. J. Lu, E. Zaccarelli, F. Ciulla, A. B. Schofield, F. Sciortino and D. A. Weitz, Nature, 2008, 453, 499-503.

6 U. Gasser, E. R. Weeks, A. Schofield, P. N. Pusey and D. A. Weitz, Science, 2001, 292, 258-262.

7 J. R. Savage and A. D. Dinsmore, Physical Review Letters, 2009, 102, 198302.

8 J. C. Crocker, Science, 2010, 327, 535-536.

9 G. Meng, N. Arkus, M. P. Brenner and V. N. Manoharan, Science, 2010, 327, 560-563.

10 N. Arkus, V. N. Manoharan and M. P. Brenner, Physical Review Letters, 2009, 103, 118303.

11 N. Arkus, V. N. Manoharan and M. P. Brenner, SIAM Journal on Discrete Mathematics, 2011, 25, 1860-1901.

12 D. J. Wales, ChemPhysChem, 2010, 11, 2491-2494.

13 R. S. Hoy, J. Harwayne-Gidansky and C. S. O'Hern, Physical Review E, 2012, 85, 051403.

14 R. S. Hoy and C. S. O'Hern, Physical Review Letters, 2010, 105, 068001.

15 F. Calvo, J. P. K. Doye and D. J. Wales, Nanoscale, 2012, 4, 1085-1100.

16 S. Asakura and F. Oosawa, Journal of Chemical Physics, 1954, 22, 1255-1256.

17 A. Vrij, Pure and Appled Chemistry, 1976, 48, 471-483.

18 G. A. Vliegenthart and P. van der Schoot, Europhysics Letters, 2003, 62, 600-606.

19 T. Biben, P. Bladon and D. Frenkel, Journal of Physics: Condensed Matter, 1996, 8, 1079910821.

20 X. Wu, R. H. Pelton, A. E. Hamielec, D. R. Woods and W. Mcphee, Colloid and Polymer Science, 1994, 272, 467-477.

21 M. Andersson and S. L. Maunu, Journal of Polymer Science Part B-Polymer Physics, 2006, 44, 3305-3314.

22 T. D. Iracki, D. J. Beltran-Villegas, S. L. Eichmann and M. A. Bevan, Langmuir, 2010, 26, 18710-18717.

23 D. B. Weibel, W. R. DiLuzio and G. M. Whitesides, Nature Reviews Microbiology, 2007, 5, 209-218.

24 A. D. Dinsmore, A. G. Yodh and D. J. Pine, Nature, 1996, 383, 239-242.

25 S. Lee, Y. Roichman, G. Yi, S. Kim, S. Yang, A. van Blaaderen, P. van Oostrum and D. G. Grier, Optics Express, 2007, 15, 18275-18282.

26 S. Lee and D. G. Grier, Optics Express, 2007, 15, 1505-1512.

27 J. Fung, K. E. Martin, R. W. Perry, D. M. Kaz, R. McGorty and V. N. Manoharan, Optics Express, 2011, 19, 8051-8065.

28 J. Fung, R. W. Perry, T. G. Dimiduk and V. N. Manoharan, Journal of Quantitative Spectroscopy and Radiative Transfer, 2012, 113, 2482-2489.

29 D. W. Mackowski and M. I. Mishchenko, Journal of the Optical Society of America A, 1996, 13, 2266-2278.

30 S. N. Kasarova, N. G. Sultanova, C. D. Ivanov and I. D. Nikolov, Optical Materials, 2007, 29, 1481-1490.

31 J. Sheng, E. Malkiel and J. Katz, Applied Optics, 2006, 45, 3893-3901.

32 L. Dixon, F. C. Cheong and D. G. Grier, Optics Express, 2011, 19, 16410-16417.

33 R. G. Newcombe, Statistics in Medicine, 1998, 17, 857-872.

34 J. C. Crocker, J. A. Matteo, A. D. Dinsmore and A. G. Yodh, Physical Review Letters, 1999, 82, 4352-4355.

35 D. R. Nelson and F. Spaepen, Solid State Physics, 1989, 42, 1-90.

36 T. A. Witten and L. M. Sander, Physical Review Letters, 1981, 47, 1400-1403.

37 J. C. Crocker and D. G. Grier, Journal of Colloid and Interface Science, 1996, 179, 298-310.

38 N. Sloane, R. Hardin, T. Duff and J. Conway, Discrete \& Computational Geometry, 1995, 14, 237-259.

39 W. B. Rogers and J. C. Crocker, Proceedings of the National Academy of Sciences, 2011, 108, $15687-15692$.

Accepted version of Faraday Disc. 113(18): 2482 (2012) 